\title{
Automatic letter priming in an alphabetic decision task
}

\author{
ARTHUR M. JACOBS and JONATHAN GRAINGER \\ Laboratoire de Psychologie Expérimentale, CNRS, Université René Descartes, Paris, France
}

\begin{abstract}
An alphabetic decision task was used to study effects of form priming on letter recognition at very short prime durations $(20$ to $80 \mathrm{msec}$ ). The task required subjects to decide whether a stimulus was a letter or a nonletter. Experiment 1 showed clear facilitatory effects of primes being either physically or nominally identical to the targets, with a stable advantage for the former. Experiment 2 demonstrated that uppercase letters are classified more rapidly as letters (vs. nonletters) when they are preceded by a briefly exposed, forward- and backward-masked, visually similar uppercase letter than when they are preceded by a visually dissimilar uppercase letter. Finally, Experiment 3 demonstrated that nominally identical and visually similar primes facilitate processing more than do nominally identical, visually dissimilar primes. The alphabetic decision task proved to produce sensitive and stable priming effects at the feature, letter, and response-choice level. The present results on letter-letter priming thus constitute a solid data base against which to evaluate other priming effects, such as word-letter priming. The results are discussed in light of current activation models of letter and word recognition and are compared with data simulated by the interactive activation model (McClelland \& Rumelhart, 1981).
\end{abstract}

The present study is concerned with the nature and time course of priming effects in a new experimental paradigm, the alphabetic decision task, which presents a promising method for the study of letter recognition (Greenberg \& Vellutino, 1988). This paradigm is analogous to the wellknown lexical decision task, probably the most widely used task in reading research. However, instead of classifying a letter string as either a word or a nonword, here subjects are required to classify a character as either a letter or a nonletter. We combined this task with a maskedpriming paradigm, as used in recent psycholinguistic experiments to study effects of form priming (Forster, 1987).

One methodological aim of the present study was to find out whether this combined paradigm is appropriate for studying questions related to effects of similarity relations (visual and/or nominal) in the mental alphabet on letter recognition. A second, theoretical aim was to answer three questions about letter recognition related to current cascaded, spreading activation models of human information processing in general (Eriksen \& Schultz, 1979; McClelland, 1979) and letter and word recognition in particular (McClelland \& Rumelhart, 1981; Paap, Newsome, McDonald, \& Schvaneveldt, 1982). Experiment 1 was designed to examine whether, for very short prime durations, one would obtain priming effects due to feature and

\footnotetext{
We wish to thank Lester Krueger, Robert Proctor, and Ken Paap for their valuable suggestions concerning improvements of an earlier version of this paper. We are also indebted to Fabrice Parpaillon for his expertise in computer programming. Correspondence should be addressed to Arthur M. Jacobs, Laboratoire de Psychologie Expérimentale, 28 rue Serpente, F-75006 Paris, France. E-mail: upec010aFRORS31.bitnet.
}

name compatibility between prime and target. Feature compatibility refers to conditions in which prime and target share some (or all) visual features; name compatibility refers to conditions in which prime and target are nominally identical but have different visual features (i.e., lower- and uppercase versions of a given letter). Experiment 2 examined whether there is a differential effect of feature compatibility, that is, whether primes facilitate target recognition to a greater extent when they share more features with the target. Finally, Experiment 3 studied the question of whether or not there is summation of priming effects due to feature and name compatibility.

In the well-studied lexical decision paradigm, wordword priming effects have been demonstrated in a number of studies for both long and short stimulus onset asynchronies (SOAs) (e.g., Colombo, 1986; Forster, 1987; Forster \& Davis, 1984; Segui \& Grainger, 1990). These effects lend themselves to an interpretation in terms of spreading activation models, such as the interactive activation model (McClelland \& Rumelhart, 1981) or the activation-verification model (Paap et al., 1982): the prior presentation of a word will affect the activation levels of orthographically related words. In an alphabetic decision task, word-letter priming was demonstrated recently using long SOAs of $180 \mathrm{msec}$ (Greenberg \& Vellutino, 1988). However, this priming effect seemed to depend on strategic factors, presumably due to the long SOA (Greenberg, 1988). Another recent study obtained evidence for word-letter priming when using a forward-mask (500 $\mathrm{msec})$, short-SOA (64 msec) paradigm (Grainger \& Jacobs, 1990). The latter study also produced clear evidence for letter-letter priming in the same experimental conditions. The prior presentation of the same letter was 
found to facilitate its subsequent processing in the alphabetic decision task. This facilitatory effect was strongest when the letters were physically identical (PI condition, prime and stimulus are presented in the same case). A significant facilitation was also obtained with masked primes presented in different case (same-name, or SN, condition). The latter result suggested that the use of a 64-msec SOA was sufficient for an abstract letter representation (presumably, but not necessarily, phonological) to be activated such that processing of the subsequent stimulus was speeded.

A general theoretical explanation for same-name priming effects is provided by the multiple-code theory of visual form perception (Posner, 1978) or similar models making use of the same basic idea. Two recent models in the tradition of Selfridge's pandemonium model for pattern recognition (Selfridge \& Neisser, 1960) and Morton's (1969) logogen model for word recognition (McClelland \& Rumelhart, 1981; Paap et al., 1982) assume that the presentation of letter stimuli activates a set of feature detectors (visual or physical code), which, in parallel, activate compatible letter detectors (name code or phonological code). The letter detectors activate word units in the mental lexicon compatible with them. In both models the name code activation is a direct continuous function of the activation pattern at the level of feature units (physical code). These activation models allow us to make a strong prediction about the relationship between the two codes: The more a prime preactivates the physical code of a letter (i.e., a specific activation pattern at the level of the feature detectors), the more its corresponding name code should be preactivated.

A convenient way to summarize hypotheses about the letter-priming effects studied here is shown in the matrix given in Table 1. It illustrates the different experimental conditions examined in Experiments 1-3. The idea is to split up the relationship between prime and target into different levels of compatibility corresponding to the hypothetical processing levels included in current models of letter and word recognition. At the feature level, we distinguish a global and a local category to separate situations in which prime and target are similar in overall shape from those in which they are form-identical. Together with the other two codes (name and response), the visual code thus allows us to obtain a maximum compatibility ranking of 4 between prime and target. This is only the case for the PI condition. On the basis of these rank values in the rightmost column of Table 1, the matrix qualitatively predicts the following response time (RT) order, $\mathrm{PI}<\mathrm{S} / \mathrm{SN}<\mathrm{D} / \mathrm{SN}=\mathrm{SIM}<\mathrm{DIS}<\mathrm{NL}$, which will be tested in this article. There are two cases, not tested here but in another report, in which the total sum corresponds to 2 . Since it is qualitative, the matrix predicts no difference in priming effects for D/SN versus SIM, that is, global feature incompatibility plus name compatibility versus global feature compatibility plus name incompatibility.

\section{EXPERIMENT 1}

In Experiment 1, we were interested in determining the prime duration for which masked-priming effects appeared. It was unclear whether a prime exposed for only $20 \mathrm{msec}$, preceded by a 500-msec forward mask and followed by a 20 -msec backward mask, would have any effect at all in this paradigm. Moreover, if the physical code were, on the average, elaborated earlier than the name code (Alwitt, 1974; Posner, 1978), PI priming effects (e.g., BB) might appear at shorter SOAs than SN priming (e.g., bB). Finally, on the basis of results from a letternaming task (Eichelman, 1970), as well as recent results from a masked-priming alphabetic decision task (Grainger \& Jacobs, 1990), we expected PI primes to yield generally shorter RTs than SN primes, both yielding shorter RTs than the two control conditions, which were different letter primes (DI condition; e.g., XB) and a blank character as prime (BL condition; e.g., "“ 'B).

\section{Method}

Subjects. Three well-trained subjects, all members of the experimental psychology lab in Paris, participated in Experiment 1, together with a fourth, untrained subject. Two of the well-trained subjects were the authors. All subjects had normal or corrected-tonormal vision.

Design and Stimuli. The target letters were 10 consonants presented in either upper- or lowercase (B, C, D, F, G, H, J, K, $\mathrm{L}, \mathrm{M})$; the character foils were 10 keyboard characters $[\%, \mathfrak{E},:$, $*,+, ?,=,),<,>]$ similar to those used by Greenberg and Vellutino (1988) and Grainger and Jacobs (1990). Target letters were preceded by four different prime conditions: (1) PI, the prime

Table 1

Priming Matrix Indicating the Theoretical Size of Letter-Priming Effects as a Function of the Compatibility Relations Between Prime and Target

\begin{tabular}{cccccc}
\hline Condition & $\begin{array}{c}\text { Global Visual } \\
\text { Features }\end{array}$ & $\begin{array}{c}\text { Local Visual } \\
\text { Features }\end{array}$ & $\begin{array}{c}\text { Abstract (Name) } \\
\text { Code }\end{array}$ & $\begin{array}{c}\text { Response } \\
\text { Code }\end{array}$ & Sum \\
\hline PI & + & + & + & + & 4 \\
S/SN & + & - & + & + & 3 \\
D/SN & - & - & - & + & 2 \\
SIM & + & - & - & + & 2 \\
DIS & - & - & - & - & 1 \\
NL & - & & - & - & 0 \\
\hline
\end{tabular}

Note-PI = physically identical (e.g., CC); S/SN = similar/same name (e.g., cC); D/SN = dissimilar/same name (e.g., gG); SIM = similar (e.g., CG); DIS = dissimilar (e.g., XG); NL = nonletter (e.g., \%G); + = compatible; $-=$ incompatible. 
was physically identical to the target (e.g., BB); (2) SN, the prime had the same name as the target but was presented in the opposite case (e.g., bB or Bb); (3) DI, the prime was a different letter from the target and not a member of the target set (e.g., XB; the following 10 consonants [either upper- or lowercase] were used: $N, P, Q$, R, S, T, V, W, X, Y); (4) BL, the prime was a blank character (". "). The character foils were preceded by the same primes. Each of the 10 target letters and character foils was presented 12 times (targets: 6 in uppercase and 6 in lowercase) in each of the four priming conditions, giving a total of 240 trials per block and subject. Four different prime durations were used: $20,40,60$, and $80 \mathrm{msec}$, corresponding to $1,2,3$, and 4 frame durations, respectively. The prime-type factor was varied within blocks; the prime-duration factor was blocked, the order being counterbalanced within each subject in a Latin square design. That is, each subject cycled through the four blocks of 240 trials four times, but in a different order and on different days. Thus, the total number of trials per subject was 3,840 .

Apparatus and Procedure. Stimulus presentation and response measurements were controlled by an Acorn-BBC Master microcomputer, using a green monitor with a $50-\mathrm{Hz}$ refresh rate. The letters corresponded to the type font "Mode 4" of the Master computer. Each letter was about $.4 \mathrm{~cm}\left(.45^{\circ}\right)$ high and $.3 \mathrm{~cm}\left(.35^{\circ}\right)$ wide. Viewing distance was maintained constant at $50 \mathrm{~cm}$. Stimuli were presented at a high contrast under photopic viewing conditions. The sequence of events on each trial was as follows. A pattern mask (\#) was presented on the center of the screen for 500 msec. This was immediately replaced in the same position by the prime stimulus, which remained on the screen for a variable duration (20, 40,60 , or $80 \mathrm{msec}$ ). This disappeared to be replaced by a backward mask (\#), remaining on the screen for one frame duration $(20 \mathrm{msec})$. Immediately after this, the target letter appeared, positioned one line lower. The target remained on the screen until the subject responded by pressing one of two buttons with the index finger of the left or right hand to indicate if the target was a letter (pressing the / key) or not (pressing the $\mathrm{Z}$ key). The subject was asked to fixate the pattern mask each time it appeared and then to respond as quickly as possible to the target appearing one line below. The RT was measured from target onset to the subject's response.

\section{Results}

Mean correct RT and errors for each experimental condition are given in Table 2 . The RTs exceeding $800 \mathrm{msec}$ were excluded from analysis (less than $1 \%$ of the data). There was a main effect of prime type on $\mathrm{RT}[F(3,9)=$
$48.19, p<.001$, but no reliable effect of prime duration $[F(3,9)=.664$, n.s.]. The interaction between the two factors was significant $[F(9,27)=2.54, p<.05]$. This indicates that priming effects increased slightly with prime duration. As confirmed by the results of a plannedcomparisons analysis, RT was shorter for letters preceded by identical letters (PI) than was RT for letters preceded by their opposite-case version $(\mathrm{SN})[F(1,3)=11.65$, $p<.05$ ], which in turn yielded reliably shorter $\mathrm{RT}$ than did letters primed by a different letter (DI) $[F(1,3)=$ $38.75, p<.01$ ]. Finally, DI-primed letters were responded to faster than were letters primed by a blank character $(\mathrm{BL})[F(1,3)=12.67, p<.05]$. An ANOVA carried out separately for the shortest prime duration $(20 \mathrm{msec}$ ) indicated that the main effect of prime type was still significant $[F(3,9)=15.47, p<.001]$.

For error rates, the only significant effect was that of prime type $[F(3,9)=28.86, p<.001]$. Errors in the SN condition exceeded those in the PI condition; however, this difference was not significant $[F(1,3)=4.96]$. Significantly more errors were made in the DI condition than in the $S N$ condition $[F(1,3)=63.44, p<.005]$. Also, significantly more errors were made in the $\mathrm{BL}$ condition than in the DI condition $[F(1,3)=34.45, p<.01]$.

As shown in Table 2, RT to nonletters was longer when these were preceded by letters ( $\mathrm{L} / \mathrm{NL}$ condition) than when they were preceded by a blank space (BL/NL). This 37 msec difference was significant $[F(1,3)=33.99$, $p<.025]$. The subjects also made significantly more errors in the former condition than they did in the latter $[F(1,3)=49.99, p<.01]$.

\section{Discussion}

Using a psychophysical approach with few subjects and many trials, the results of Experiment 1 replicate our recent finding that, in a masked-priming alphabetic decision task, PI primes yield more RT facilitation than do SN primes (Grainger \& Jacobs, 1990, Experiment 2). The new aspect is that, in such a forward/backward masking paradigm, even for short prime durations we obtain both form- and name-priming effects with regard to the two

Table 2

Mean Response Time (RT; in milliseconds) and Percentage of Error (PE) Rate $\times$ Prime Type and Prime Duration

\begin{tabular}{|c|c|c|c|c|c|c|c|c|c|c|}
\hline \multirow{3}{*}{$\begin{array}{l}\text { Prime } \\
\text { Type }\end{array}$} & \multicolumn{8}{|c|}{ Prime Duration (msec) } & \multirow[b]{3}{*}{ \% Facilitation } & \multirow[b]{3}{*}{ \% Inhibition } \\
\hline & \multicolumn{2}{|c|}{20} & \multicolumn{2}{|c|}{40} & \multicolumn{2}{|c|}{60} & \multicolumn{2}{|c|}{80} & & \\
\hline & RT & $\overline{\mathrm{PE}}$ & $\overline{\mathrm{RT}}$ & $\overline{\mathrm{PE}}$ & $\overline{\mathrm{RT}}$ & $\mathrm{PE}$ & RT & PE & & \\
\hline PI & 381 & 5.3 & 378 & 3.4 & 371 & 2.5 & 366 & 0.9 & 9.4 & - \\
\hline SN & 389 & 5.3 & 387 & 5.6 & 385 & 3.7 & 375 & 3.4 & 7.0 & 2.6 \\
\hline DI & 406 & 10.0 & 404 & 8.7 & 401 & 9.7 & 399 & 11.4 & 2.6 & 7.4 \\
\hline $\mathrm{BL}$ & 414 & 15.4 & 410 & 18.4 & 411 & 15.9 & 416 & 20.7 & - & 10.4 \\
\hline BL/NL & 386 & 0.9 & 385 & 1.4 & 386 & 1.0 & 385 & 0.9 & 6.5 & - \\
\hline $\mathrm{L} / \mathrm{NL}$ & 413 & 11.4 & 423 & 12.0 & 426 & 15.0 & 428 & 17.9 & - & 6.9 \\
\hline
\end{tabular}

Note-PI = physically identical (e.g., BB); SN = same name (e.g., bB); DI = different letter (e.g., XB); $\mathrm{BL}=$ blank space/letter; BL/NL = blank/nonletter; $\mathrm{L} / \mathrm{NL}=$ letter/nonletter (e.g., g\%). The last two columns give the percentage of RT facilitation ( $\%$ Facilitation) and of RT inhibition ( $\%$ Inhibition) for each condition, relative to the two baseline conditions, marked by a - In regard to the percentage of facilitation for letters, the control condition was BL-for example, percentage of PI facilitation $=[R T(B L)-R T(P D / R T(B L)]$. In regard to the percentage of inhibition for letters, the baseline condition was PI. 
control conditions (DI and BL). The size of the priming effect increases only slightly with prime duration, and, at least for the present durations, no evidence was obtained that PI-priming effects occur at shorter prime durations than do SN priming effects.

Within the theoretical context discussed in the introduction, our data indicate that masked letter primes activate both physical and abstract (name) codes in an alphabetic decision task even when they are exposed as briefly as $20 \mathrm{msec}$. Since PI primes yield maximum facilitation, we can assume that the combined activation of units processing physical and abstract features is more efficient for determining whether or not a given stimulus is a letter than is the activation of abstract units only (i.e., in the SN condition).

The PI- and SN-priming effects, which are compatible with what can be expected from the priming matrix given in Table 1 , can be readily explained by current activation models of letter and word recognition. More specifically, according to the interactive activation model (McClelland \& Rumelhart, 1981), in the PI condition, the units mediating recognition of the target letter start from a relatively high activation level, because both the feature units and the abstract unit compatible with the target have received preactivation by the prime. Since the available implemented version of the interactive activation model (McClelland \& Rumelhart, 1988) includes no lowercase letters, strictly speaking, the model cannot be used to account for SN-priming effects. However, conceptually at least, it is easy to adapt the model in such a way as to be able to deal also with SN priming. Thus, in the SN condition, the feature units compatible with the subsequent target letter should have been inhibited by the prime, at least to a certain extent, and only the abstract unit would have received preactivation.

The SN-over-DI advantage, already present at a 20 msec prime duration, has also been observed in a letternaming study (Eichelman, 1970) and a same/different matching paradigm (Proctor, 1981). It has been explained by the mutual inhibition of internal recognition responses (Proctor, 1981). Thus, in the DI condition, the prime activates its corresponding name code and the target activates its own. In contrast to the $\mathrm{SN}$ condition, in which only one name code is activated, in the DI condition both codes compete, yielding longer RT. The interpretation in terms of the interactive activation model is compatible with this. The DI prime inhibits both feature and abstract (name) units compatible with the subsequent target, but incompatible with the prime. Thus, in the DI condition, the units processing the target start from (negative) values below their normal resting level activations. In contrast, in the SN condition, the abstract unit processing the target has already received preexcitation by the nominally identical prime, thus reaching its activation asymptote earlier than in the DI condition.

A finding that merits discussion, and one that is harder to implement in the interactive activation model, is the obtained different-letter-over-blank-character (i.e., DIprime-over-BL-prime) advantage. One might think that a DI prime inhibits the perception of the subsequent (different) letter more than does a blank character that, a priori, should neither activate nor inhibit any of the hypothetical feature and letter units supposed to mediate letter recognition in the model. Thus, the present finding could be interpreted as indicating that DI primes did not inhibit target processing in this alphabetic decision task. In fact, with regard to blank characters, they produced about $3 \%$ facilitation.

To reconcile this finding with the interactive activation model, one possibility is to consider that, on a nonnegligible proportion of trials, the DI primes, although different, were visually similar to the stimulus letter, thus leading to some facilitation (at the level of feature coding). We did not control for visual similarity between primes and targets in Experiment 1. Thus, for example, the DI primes $\mathrm{N}$ or $\mathrm{n}$ might well have facilitated processing of the targets $\mathbf{M}$ or $\mathrm{m}$, respectively. If, on the majority of trials, DI primes were visually similar to the target and thus facilitated its processing, then DI primes should have led to shorter RT than the neutral BL primes.

Another possibility for explaining the above finding (DIover-BL advantage) is in terms of competition at the level of response preparation and choice (Eriksen \& Schultz, 1979). Thus, one might think that, in this task, the subjects developed a stable association between letters and a right-hand response and between nonletters and a lefthand response. If blanks are neutral at the response-bias level, whereas letter primes might produce a bias toward a letter response in the DI condition, then the longer RTs and more errors in the BL condition could be seen as the result of a response bias. The results concerning RT and error rates for nonletter targets support this interpretation: When nonletter targets were preceded by letter targets, mean RT was almost $40 \mathrm{msec}$ longer and about $14 \%$ more errors were made than when they were preceded by blanks. This issue is further addressed in Experiment 3.

In fact, the blank character does not seem to be an optimal control condition, since presumably it requires no processing at all. We therefore used another control condition in Experiment 2, presenting a nonletter character as prime (the ${ }^{*}$ ).

\section{EXPERIMENT 2}

Experiment 2 tested whether different, visually similar primes facilitate target processing (e.g., QO), as compared with different, dissimilar primes (e.g., TO) in a masked-priming alphabetic decision task at a short, 60msec prime duration. The data should allow us to conclude whether or not feature compatibility between prime and target facilitates target processing in conditions of name incompatibility, as expected from the priming matrix given in Table 1 and as predicted by current activation models of letter and word recognition.

\section{Method}

Subjects. Ten 3rd-year students of experimental psychology from René Descartes University in Paris and 10 members of the ex- 
perimental psychology lab, very familiar with RT studies, participated in Experiment 2.

Design and Stimuli. A simple one-factor, repeated measures design was used, in which the factor was priming condition. The target stimuli were 10 uppercase letters $(C, E, F, G, I, O, Q, T, U$, $V)$. These letters allowed the formation of five visually similar pairs (prime and target): CG, EF, IT, $O Q$, and UV. Dissimilar pairs were obtained by combining letters from different pairs (e.g., IC or VT). The character foils were 10 keyboard characters $[\%, \mathfrak{£},:, \$,+$, $?,=,),<,>$ ]. Target letters were presented in uppercase and preceded by four different prime conditions: (1) PI, the prime was physically identical to the target (e.g., OO); (2) SIM, the prime was visually similar to the target (e.g., QO); (3) DIS, the prime was visually dissimilar to the target (e.g., IO); (4) NE, the prime was a neutral character (the ${ }^{*}$ ). The character foils were preceded by the same primes. Each of the 10 target letters and 10 character foils was presented twice in each of the four priming conditions, giving a total of 160 trials per experimental session and subject.

Apparatus and Procedure. In Experiment 2, we used the same apparatus as that used in Experiment 1 . The sequence of events on each trial was as follows. A checkerboard mask, which covered an area of a full letter matrix, was presented on the center of the screen for $500 \mathrm{msec}$. This was immediately replaced in the same position by the prime stimulus, which remained on the screen for $60 \mathrm{msec}$. This was replaced by a checkerboard backward mask, which remained on for one frame duration $(20 \mathrm{msec})$. The checkerboard mask was used rather than the hash-mark mask of Experiment 1 , because the checkerboard is more neutral with regard to letter features (e.g., horizontal and vertical bars) than is the hash mark, and because the checkerboard proved to be very efficient in a related experiment on letter priming in a character-classification task. Although we have no evidence for it, the hash mark might have differential masking effects depending on whether or not the prime and/or the target have diagonal features.

Immediately after the backward mask, the target letter appeared in the same position and on the same line as prime and mask. Several word-letter and letter-letter priming studies done in our laboratory have shown that, with the present paradigm and conditions, the two presentation modes (with the target one line below the prime vs. the target in same line) yield identical results (Grainger \& Jacobs, 1990). The latter mode was chosen here because it does not require subjects to move their gaze continually between two lines and thus avoids possible problems due to shifts of visual attention and eye-movement programming. The target remained on the screen until the subject responded by pressing one of two buttons. The subjects were asked to fixate the pattern mask each time it appeared and to respond as quickly as possible to the target.

\section{Results and Discussion}

As shown in Table 3, RT for correct letter classification increased when prime-target compatibility (as indicated in Table 1) decreased from the PI condition to the NE condition $[F(3,57)=106.96, p<.001]$. Planned comparisons confirmed that RT to PI-primed letters (e.g., OO) was shorter than RT to SIM-primed letters (e.g., QO) $[F(1,19)=25.35, p<.001]$. SIM primes led to reliably shorter RT than did DIS primes (e.g., TO) $[F(1,19)$ $=11.08, p<.005$ ]. Finally, DIS primes yielded shorter RT than did NE primes (i.e., the neutral * $[F(1,19)=$ $72.53, p<.001]$. The error rates indicated no sign of a speed-error tradeoff. In regard to the amount of facilitation, using a different dissimilar letter (DIS), rather than a nonletter prime (NE), yielded about $15 \%$ RT facilitation. When the prime was similar (SIM), $18 \%$ was ob-
Table 3

Mean Response Time (RT; in milliseconds) and Percentage of Error (PE) Rate $\times$ Prime Type

\begin{tabular}{lcccc}
\hline $\begin{array}{l}\text { Prime } \\
\text { Type }\end{array}$ & RT & PE & \% Facilitation & \% Inhibition \\
\hline PI & 367 & 3.13 & 23.8 & - \\
SIM & 395 & 4.03 & 18.0 & 7.6 \\
DIS & 412 & 2.97 & 14.5 & 12.2 \\
NE & 482 & 16.3 & - & 31.3 \\
NE/NL & 397 & 2.00 & 13.3 & - \\
L/NL & 458 & 9.66 & - & 15.3 \\
\hline
\end{tabular}

Note-PI = physically identical (e.g., OO); SIM = similar letter (e.g., QO); DIS = dissimilar letter (e.g., TO); NE = neutral (e.g., "O); $\mathrm{NE} / \mathrm{NL}=$ neutral/nonletter (e.g. . $* \%) ; \mathrm{L} / \mathrm{NL}=$ letter/nonletter (e.g. . T\%); $\%$ Facilitation $=$ percentage of RT facilitation; $\%$ Inhibition $=$ percentage of RT inhibition; - = baseline condition.

tained, compared with a maximum of about $24 \%$ for the identity-priming (PI) condition.

The data are compatible with what could be expected from the priming matrix of Table 1. Uppercase letters are classified more rapidly as letters (vs. nonletters) when they are preceded by a briefly exposed, forward- and backward-masked, visually similar uppercase letter than when they are preceded by a visually dissimilar uppercase letter. According to the interactive activation model (McClelland \& Rumelhart, 1981), the prime Q, say, would preactivate a high percentage of the feature units compatible with the target letter $O$. Simultaneously, it would preactivate the abstract $Q$ unit and, albeit to a lesser degree, the abstract $O$ unit. A prime visually dissimilar to the $O$, say the $T$, would not only lead to strong inhibition at the name code level, but also strongly inhibit target processing at the level of feature extraction.

The strongest difference in RT in Table 3 is the one between dissimilar letter primes and neutral primes (the * character). To account for this finding within the framework of the interactive activation model, one would have to assume that the * prime strongly inhibits all feature and letter units of the mental alphabet. However, an alternative and presumably more plausible way of explaining this effect is in terms of response competition. The long RT and high error rate in the NE condition support the view that, whenever a nonletter precedes a letter, this yields inhibition at the level of response choice, whereas letters (whether the same as, name-identical, similar, or dissimilar to the target) generally do not. This sort of response-category priming might represent a global mechanism that can be overriden by the subtler effects of local form/similarity or name priming. If this were true, however, one should also find that RT to a nonletter primed by another nonletter is shorter than RT to a nonletter primed by a letter. The data concerning nonletters in Table 3 clearly suggest this, since $R T$ is longer and error rate higher in the $\mathrm{L} / \mathrm{NL}$ condition than in the NE/NL condition.

As a more thorough test of the interactive activation model, its available implemented version (McClelland \& 
Rumelhart, 1988) was used to simulate the data of Experiment 2 . The details and results of this simulation are given in the Appendix. A linear regression analysis using a least squares criterion provided estimates of the duration of each processing cycle of the model for the present task and conditions. These estimates were then used to predict RT in each condition according to either an activation-based (ACT) or a probability-based (PROB) response criterion. The RTs predicted by the ACT and PROB simulations fit the observed RTs about equally well. However, the intercept of the regression equation, reflecting components of processing not simulated by the model (i.e., sensorimotor time) has a more reasonable value for ACT simulation (224.8 msec) than for PROB simulation $(-58 \mathrm{msec})$. Simulations using different response criteria $(50 \%, 70 \%$, and $90 \%$ of the asymptotic activation or probability) did not yield better fits than did the $95 \%$ criterion chosen originally. Other studies using more than the present three data points are necessary to decide which response criterion is most appropriate to simulate binary RTs in this task. In any case, the simulation results presented in the Appendix demonstrate that a version of the interactive activation model in which no changes were made with regard to the original parameter values of the computer program provided in McClelland and Rumelhart (1988) can be used to predict the empirical results concerning RT to primed uppercase letters. The model describes quite well the RT rank order obtained in the present alphabetic decision task, as well as the finding that the difference between the PI condition and the SIM condition is greater than that between the SIM condition and the DIS condition.

\section{EXPERIMENT 3}

Apart from a direct test of the category-priming/ response-competition hypothesis, for which indirect evidence was obtained in Experiments 1 and 2, a second issue was investigated in Experiment 3. It concerned a possible summation of feature- and name-compatibility effects. According to the priming matrix shown in Table 1, a prime that is both feature and name compatible with the target should yield stronger facilitation than should one that is only name compatible. Therefore, trials were included in which the lower- and uppercase versions of the target letter were visually similar (e.g., c C, k K, o O, p P, s S, u U, v V, w W, x X, z Z) and in which they were not (e.g., a A, b B, d D, e E, f F, g G, i I, j J, $q \mathrm{Q}, \mathrm{r} R$ ).

\section{Method}

Subjects. Twenty-five 3rd-year students of psychology from René Descartes University participated as subjects in this experiment for course credit.

Apparatus and Procedure. The apparatus and procedure used in Experiment 3 were identical to those used in Experiment 2.

Stimuli. The following target stimuli were used: uppercase letters with similar lowercase versions (C, K, O, P, S, U, V, W, X, $Z$ ), uppercase letters with dissimilar lowercase versions $(A, B, D$, $\mathrm{E}, \mathrm{F}, \mathrm{G}, \mathrm{I}, \mathrm{J}, \mathrm{Q}, \mathrm{R})$, and nonletters $(!, \$, \%, \&,+, *, ?, \#,<$,
>). As in Experiment 2, a checkerboard stimulus was used as forward and backward mask. Target letters were presented in uppercase. Due to the use of nonletter targets, seven different priming conditions were tested: (1) PIL, the prime was physically identical to the target letter (e.g., CC); (2) PINL, the target and the prime were physically identical nonletters (e.g., \$\$); (3) S/SN, the prime was visually similar to the target letter and had the same name (e.g., cC); (4) D/SN, the prime was visually dissimilar to the target letter and had the same name (e.g., aA); (5) DINL, the prime was a nonletter different from the nonletter target (e.g., \&\$); (6) L/NL, the prime was a letter and the target was a nonletter (e.g., A\$); (7) NL/L, the inverse of L/NL (e.g., \$A). To equilibrate prime and target, letter and nonletter frequencies, the target letters were presented 20 times in the PIL condition, 10 times each in the S/SN and $\mathrm{D} / \mathrm{SN}$ conditions, and 40 times in the NL/L condition. The nonletter targets were presented 20 times each in the PINL and DINL conditions and 40 times in the L/NL condition. This yielded a total of 160 trials per experimental block. Each subject cycled through two blocks per session. Block number was considered an experimental factor in order to evaluate possible training effects. Half of the subjects responded to letters with their right index fingers and to nonletter targets with their left index fingers. Response assignment was reversed for the other half.

\section{Results}

Table 4 gives mean RT and percent error for each experimental condition. The RTs exceeding $800 \mathrm{msec}$ were excluded from analysis (less than $3 \%$ of data). There was a main effect of prime type on $\mathrm{RT}[F(6,144)=67.81$, $p<.001]$. The block factor also yielded a slight, but significant, effect $[F(1,24)=5.19, p<.05]$. Mean RT decreased from $518 \mathrm{msec}$ in the first block to $502 \mathrm{msec}$ in the second. The prime type $\times$ block interaction was not significant. The data shown in Table 4 demonstrate that, as in the preceding two experiments, RT was shortest to uppercase letters primed by themselves (as in the PIL condition, e.g., OO), representing about $11 \%$ RT facilitation. Planned comparisons confirmed that RT was shorter here than RT to uppercase letters primed by similar lowercase versions (as in the $\mathrm{S} / \mathrm{SN}$ condition, e.g., oO) $[F(1,24)=9.7, p<.005]$. With about $8 \%$ facilitation, the latter, in turn, yielded significantly shorter RT than did uppercase letters primed by dissimilar lowercase ver-

Table 4

Mean Response Time (RT; in milliseconds) and Percentage of Error (PE) Rate $\times$ Prime Type

\begin{tabular}{|c|c|c|c|c|}
\hline $\begin{array}{c}\text { Prime } \\
\text { Type }\end{array}$ & RT & PE & $\%$ Facilitation & \% Inhibition \\
\hline PIL & 465 & 5.12 & 10.9 & - \\
\hline S/SN & 480 & 6.06 & 8.0 & 3.2 \\
\hline $\mathrm{D} / \mathrm{SN}$ & 507 & 6.77 & 2.8 & 9.0 \\
\hline NL/L & 522 & 11.3 & - & 12.2 \\
\hline PINL & 507 & 11.0 & 9.6 & - \\
\hline DINL & 529 & 7.89 & 5.7 & 4.3 \\
\hline $\mathrm{L} / \mathrm{NL}$ & 561 & 14.8 & - & 10.6 \\
\hline
\end{tabular}

Note-PIL = physically identical letter (e.g., CC); S/SN = similar/same name (e.g., cC); D/SN = dissimilar/same name (e.g., aA); NL/L = nonletter prime for letter target (e.g., \$C); PINL = physically identical nonletter (e.g., \$\$); DINL = different nonletter (e.g., \%\$); L/NL $=$ letter prime for nonletter target (e.g., A\$); \% Facilitation = percentage of RT facilitation; \% Inhibition = percentage of RT inhibition; - = baseline condition. 
sions (as in the D/SN condition, e.g., aA) $[F(1,24)=$ $48.65, p<.001]$. Here, RT facilitation was about $3 \%$. Finally, the longest RT for letter targets was obtained when the prime was a nonletter (as in the NL/L condition, e.g., \&O). This NL/L versus $\mathrm{D} / \mathrm{SN}$ difference was significant $[F(1,24)=5.52, p<.05]$.

In regard to nonletter targets, RTs were significantly shorter when they were primed by themselves (as in the PINL condition, e.g., \%\%) than when they were preceded by a different nonletter (as in the DINL condition, e.g., $\$ \%)[F(1,24)=15.8, p<.005]$. The RT to nonletter targets was longer when they were preceded by letters (L/NL condition) than when they were preceded by a different nonletter (DINL condition) $[F(1,24)=106.82$, $p<.001]$.

For letter targets, the only significant difference on errors was that between the $\mathrm{D} / \mathrm{SN}$ and $\mathrm{NL} / \mathrm{L}$ conditions $[F(1,24)=29.13, p<.001]$. For nonletters, the tendency to erroneously respond "letter" was significantly greater when letter primes were used (L/NL condition) than when nonletter primes were used (PINL and DINL conditions) $[F(1,24)=5.96, p<.025]$. The somewhat puzzling tendency to respond "letter" more frequently when the nonletter was preceded by an identical nonletter shape (PINL condition) than when it was preceded by a nonidentical nonletter shape (DINL condition) was not significant $[F(1,24)=2.75]$.

Interestingly, letters preceded by nonletters yielded significantly shorter RT $(522 \mathrm{msec})$ than did nonletters preceded by letters $(561 \mathrm{msec})[F(1,24)=38.93$, $p<.001]$. This could reflect either a decisional bias in favor of letters, which, in this task, were considered the positive response, or a significant difference in stimulus familiarity, or both.

\section{Discussion}

As expected, RT was facilitated most when prime and target were identical in both form and name. The main result here is the demonstration that uppercase letters are classified more rapidly as letters (vs. nonletters) when they are preceded by a visually similar, name-identical stimulus (e.g., cC) than when they are preceded by a visually dissimilar, name-identical stimulus (e.g., gG). In terms of RT facilitation, adding (global) feature compatibility (i.e., S/SN condition) to name identity (in both S/SN and $\mathrm{D} / \mathrm{SN}$ ) increased priming by about $5 \%$. These data are compatible with the priming matrix given in Table 1 and, in principle, also with the interactive activation model. Although the model deals with only uppercase letters, it is at least conceptually easy to extend it so that the prime c, say, would preactivate a critical percentage of the feature units compatible with the target $C$. In contrast, the prime $\mathrm{g}$ would inhibit a critical percentage of the feature units compatible with the target G.

Together with the results of Experiments 1 and 2 concerning nonletter targets, the finding that the longest RT and the highest error rate for letter targets were obtained in the condition in which the target letter was preceded by a nonletter is evidence in favor of response competition and, thus, of the continuous flow model (Eriksen \& Schultz, 1979). Looking at the rightmost column of Table 4 , one can see that response competition that arose due to the fact that prime and target belonged to opposite response categories (NL/L condition) added about $6 \%$ inhibition, relative to when prime and target belonged to the same category (S/SN and D/SN conditions). Correspondingly, for nonletter targets, the longest $\mathrm{RT}$ and highest error rate were obtained when primes were letters ( $\mathrm{L} / \mathrm{NL}$ condition). The finding that there is identity priming also for nonletters (i.e., the PINL-over-DINL advantage) suggests that, at least for the present keyboard characters (e.g., \%, \$, or ?), subjects possess some kind of internal representation of nonletters. If this is the case, one would not expect to observe such priming with scrambled letter fragments as the nonletter stimuli, an easily testable prediction for a future study.

\section{GENERAL DISCUSSION}

The present study examined letter-priming effects for very short prime durations in an alphabetic decision task. This task, first used by Greenberg and Vellutino (1988), requires that subjects decide on the basis of visually presented information whether or not an isolated target stimulus belongs to the alphabet. Our results show that this experimental paradigm produces sensitive and stable priming effects for short prime durations, whether one chooses a psychophysical approach with few trained subjects (Experiment 1) or a general approach with many relatively untrained subjects (Experiments 2 and 3). Given the simplicity and versatility of the task, this is encouraging for future studies concerned with human information processing and letter and word recognition.

The data of three experiments demonstrated that, when subjects classify a target stimulus as either a letter or a nonletter, briefly presented forward- and backwardmasked prime stimuli strongly affect RTs and error rates to the targets. In all experiments, target processing was facilitated most by prior presentation of the target itself in the same case (i.e., identity priming was most efficient). Even for short prime durations not only was identity priming obtained, but also same-name priming. Together with the results on letter naming (Eichelman, 1970) and letter matching (Krueger, 1978; Posner, 1978; Proctor, 1981), these priming data provide more converging evidence for the notion that the system processing written language automatically computes several codes representing the input stimuli (presumably of a visual and phonological nature; see Carr, 1986).

In addition, form-similarity (feature-compatibility) priming was obtained for a 60 -msec prime duration (Experiment 2): prior presentation of $F$ facilitated processing of $E$, relative to $A$ before $E$, for example. Finally, also for a 60 -msec prime duration, feature compatibility plus name identity between prime and target (e.g., cC) yielded shorter RT than did feature incompatibility plus 
name identity (e.g., aA; see Experiment 3). These data nicely demonstrate how the different codes may combine during the processing of the input. They suggest that, as claimed by the interactive activation model (McClelland \& Rumelhart, 1981) and other feature-accumulation, continuous-processing models (Eriksen \& Schultz, 1979; Estes, 1975; Selfridge \& Neisser, 1960), that information flows from the feature level to abstract letter and response representations, with facilitation and inhibition accumulating between these levels. What exactly the visual features detected by these primitive representational units are remains a matter of research and debate. A priori, all visual properties, whether more global or more local, whether more physical or more abstract, are possible. These include, for example, luminance profile, size, height-to-width quotient, overall shape, oriented line segments, symmetry, and openness (see Jacobs, Nazir, \& Heller, 1989). Experiment 3 suggests that, whatever the exact features, the abstract representation of a letter (its name) is constructed more easily and/or more clearly when the two stimuli (i.e., prime and target) that activate it have similar visual features than it is when they have not.

The interactive activation model (McClelland \& Rumelhart, 1981) seems to constitute a unified implementable theoretical framework to accommodate the present results if one extends it by adding processing units for lowercase letters and a binary response-choice mechanism. According to the model, a prime whose features and name are identical to those of the target (i.e., the PI condition) will lead to a maximum of preexcitation at the level of both feature and letter nodes. On target presentation, since only facilitatory activation exists, the units start processing from a higher than normal activation level and reach asymptote more quickly. Since, in the model, a response is chosen-at the letter level-when asymptote is reached, it is obvious why the PI condition yields the shortest RT with maximum preactivation (see Figure A1). When some of the feature units responding to the target are preactivated by a prime nominally identical to the target and having a similar shape (e.g., cC), the sum of the preactivation at the feature level plus the preactivation at the abstract (name) level leads to reliably faster processing of the target than when only the abstract unit is preactivated and most or all of the feature units responding to the target are inhibited by the prime (i.e., a nominally identical prime with dissimilar shape; e.g., aA).

Similarly, when prime and target have different identities but similar shapes (e.g., QO), the amount of preactivation at the feature level is sufficient to yield faster target processing than when prime and target are both feature and name incompatible (e.g., TO), thus yielding maximum inhibition (see Figure A1). The simulation results presented in the Appendix demonstrate that the interactive activation model can be used to predict this finding without any changes in the original parameter values. It should be noted, however, that the simulation concerning the results of Experiment 2 can only be considered a partial test of the interactive activation model. A more complete test should include simulating the results concerning same-name priming (Experiment 1), as well as those concerning RT to nonletter targets, including possible effects due to response-category priming (Experiment 3). Although the model has enough structure to make it conceptually easy to incorporate the necessary modifications, such a test would, of course, require some major changes in the implementation of the model (e.g., including a set of feature detectors for lowercase letters, a binary response-choice level, and possibly some units for coding familiar nonletter stimuli). These necessary modifications clearly exceed the scope of the present paper. However, as a first step towards testing the interactive activation model in tasks other than the Reicher (1969) paradigm, for which it was originally designed, the present restricted simulations-together with those of Grainger's (1990) successful application of the model to a lexical decision task-provide quite encouraging results. Future more sophisticated tests, including a comparison of simulation results with competing, implementable models (e.g., the activation-verification model) in different paradigms will reveal whether the interactive activation model or its competitors are generally valid theoretical frameworks for understanding the process of letter and word recognition.

To improve our understanding of the letter-recognition process, one aim for future studies must be to develop the quantitative analysis of stimulus properties, as well as processing operations, to a point allowing us to fill the priming matrix (see Table 1) with interval-scale data rather than ordinal data. Furthermore, by manipulating the ISI (i.e., the interval between the prime and the target) rather than prime duration, one could study the time course of the above priming effects. Since the interactive activation model has a parameter specifying the decay rate of the processing units at all levels, it seems possible to test quantitative predictions about the critical ISI (i.e., the ISI value), beyond which a PI or SN prime should no longer facilitate target processing in the present task. Thus, one might think that gG priming would be more resistant to increasing ISI than would CG priming, given that the persistence of the abstract code of a letter or word is more important for further processing at higher levels than is that of the physical code.

The present results constitute a solid data base against which to evaluate other possible priming effects. Grainger and Jacobs (1990) have recently applied the alphabetic decision task combined with the masked priming paradigm to study word-letter priming. This area of research should help develop our understanding of the types of relations existing between word representations in memory and the representations of their component letters particularly in terms of the presence or absence of facilitatory or inhibitory feedback from the word to the letter level. Another line of research directly linked to this concerns the possible facilitatory effects of prime letters on word recognition. We are currently involved in research manipulating the various combinations of a target word's component 
letters in the masked-priming paradigm combined with the lexical decision task. The data made available by research in these key areas (letter-letter, word-letter, and letter-word priming) should help in the future refinement of models of letter and word recognition.

\section{REFERENCES}

ALWITT, L. F. (1974). Feature similarity and feature bias in identification of visually presented letters. Memory \& Cognition, 2, 5-10.

CARR, T. H. (1986). Perceiving visual language. In K. R. Boff, L. Kaufman, \& J. P. Thomas (Eds.), Handbook of perception and human performance (pp. 29.1-29.82). New York: Wiley.

Colomвo, L. (1986). Activation and inhibition with orthographically similar words. Journal of Experimental Psychology: Human Perception \& Performance, 12, 226-234.

Eichelman, W. H. (1970). Stimulus and response repetition effects for naming letters at two response-stimulus intervals. Perception \& Psychophysics, 7, 94-96.

ERIKSEN, C. W., \& SChulTZ, D. W. (1979). Information processing in visual search: A continuous flow conception and experimental results. Perception \& Psychophysics, 25, 249-263.

EsTEs, W. K. (1975). The locus of inferential and perceptual processes in letter identification. Journal of Experimental Psychology: General, 104, 122-145.

FoRster, K. I. (1987). Form priming with masked primes: The best match hypothesis. In M. Coltheart (Ed.), Attention and performance XII: The psychology of reading (pp. 127-146). Hillsdale, NJ: Erlbaum.

Forster, K. I., \& DAVIS, C. (1984). Repetition priming and frequency attenuation in lexical access. Journal of Experimental Psychology: Learning, Memory, \& Cognition, 10, 680-690.

GrAINGER, J. (1990). Word frequency and neighborhood frequency effects in lexical decision and naming. Journal of Memory \& Language, 29, 228-244.

Grainger, J., \& JAcoBs, A. M. (1990). Priming letter recognition with words, nonwords, and single letters. Manuscript submitted for publication.

Greenberg, S. N. (1988). Are letter codes always activated? Perception \& Psychophysics, 44, 331-338.

GreEnBerg, S. N., \& Vellutino, F. R. (1988). Evidence for processing of constituent single and multiletter codes: Support for multilevel in word perception. Memory \& Cognition, 16, 54-63.

JaCOBS, A. M., NAZIR, T. A., \& Heller, O. (1989). Perception of lowercase letters in peripheral vision: A letter discrimination matrix based on saccade latencies. Perception \& Psychophysics, 46, 95-102.

Krueger, L. E. (1978). A theory of perceptual matching. Psychological Review, 85, 278-304.

MCClelland, J. L. (1979). On the time relations of mental processes: An examination of systems of processes in cascade. Psychological Review, 86, 287-330.

McClelland, J. L., Rumelhart, D. E. (1981). An interactive activation model of context effects in letter perception. Part I: An account of basic findings. Psychological Review. 88, 375-407.

MCClelland, J. L., \& Rumelhart, D. E. (1988). Parallel distributed processing: Explorations in the micro-structure of cognition (Vol. 3). Cambridge, MA: Bradford Books.

Morton, J. (1969). Interaction of information in word recognition. Psychological Review, 76, 165-178.

PaAp, K. R., Newsome, S. L., McDonald, J. E., \& Schvaneveldt, R. W. (1982). An activation-verification model for letter and word recognition: The word-superiority effect. Psychological Review, 89, 573-594.

PoSNER, M. K. (1978). Chronometric explorations of the mind. Hillsdale, NJ: Erlbaum.

Proctor, R. W. (1981). A unified theory for matching task phenomena. Psychological Review, 88, 291-326.

REICHER, G. W. (1969). Perceptual recognition as a function of meaning fulness of stimulus material. Journal of Experimental Psychology, 81, 274-280.
Segui, J., \& Grainger, J. (1990). Priming word recognition with orthographic neighbors: The effects of relative prime-target frequency. Joumal of Experimental Psychology: Human Perception \& Performance, 16, 65-76.

Selfridge, O. G., Neisser, U. (1960). Pattern recognition by machine. Scientific American, 203, 60-68.

\section{APPENDIX \\ Simulations Using the Interactive Activation Model}

To adapt the interactive activation model at least partially to the present paradigm and experimental situation, two minor modifications of the original computer program were necessary. First, information flow to and from the word level was eliminated. Second, to provide a measure of letter-identification latencies, a response criterion for letter-node activation levels was

Table A1

Comparison of Observed and Predicted Response Time (RT) Data for the First Three Conditions of Experiment 2

\begin{tabular}{|c|c|c|c|c|c|}
\hline \multirow{2}{*}{$\begin{array}{c}\text { Prime } \\
\text { Type }\end{array}$} & \multicolumn{2}{|c|}{ Number of Cycles } & \multirow{2}{*}{$\begin{array}{c}\text { RT } \\
\text { Observed }\end{array}$} & \multicolumn{2}{|c|}{ RT Predicted } \\
\hline & ACT & PROB & & ACT & PROB \\
\hline PI & 19.0 & 54.8 & 367 & 365 & 364 \\
\hline SIM & 23.6 & 59.2 & 395 & 399 & 398 \\
\hline DIS & 24.6 & 60.2 & 412 & 407 & 405 \\
\hline
\end{tabular}

Note-Linear equations relating $\mathrm{RT}$ to number of processing cycles $(X)$ : For ACT, RT = 224.8 + 7.4X, $r=.977$; for PROB, RT $=-58.0$ $+7.7 X, r=.978 . \mathrm{PI}=$ physically identical (e.g., OO); $\mathrm{SIM}=$ similar letter (e.g., QO); DIS = dissimilar letter (e.g., TO). RT data is given in milliseconds.

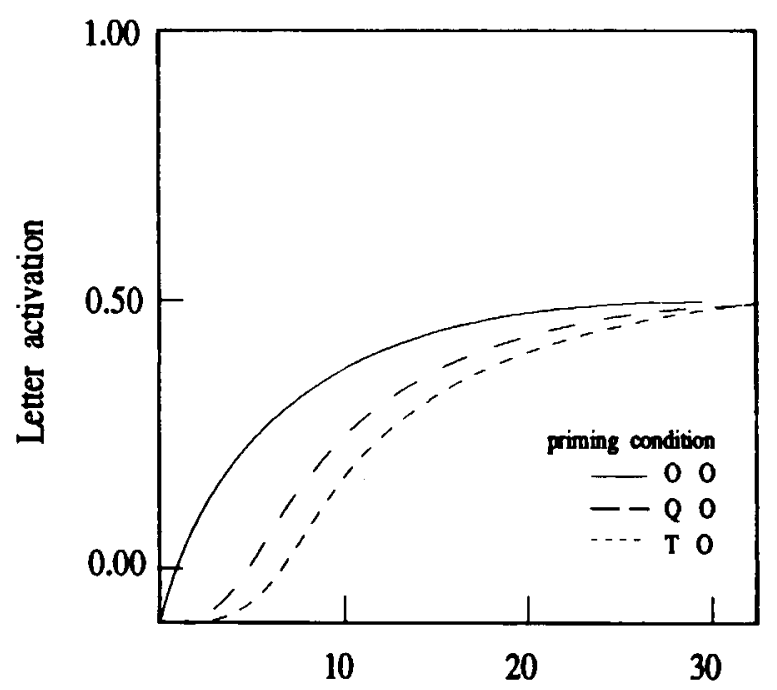

Number of processing cycles

Figure A1. The time course of activation of the node corresponding to the letter $O$ after presentation of a prime and a neutral backward mask, for three conditions: 00 (i.e., prime and target are identical), $\mathbf{Q O}$ (i.e., prime and target are visually similar), and TO (i.e., prime and target are dissimilar). Time is given in number of processing cycles relative to target onset. 
implemented in the model: The number of processing cycles corresponding to a value of $95 \%$ of either the asymptotic activation of a given letter unit (ACT simulation) or its response probability (PROB simulation) was considered to be the final response time. For the simulation reported below, nothing else in the architecture or the parameters of the original model was changed. One simulated trial consisted of presenting a prime letter at Field Time 1, a backward mask at Field Time 4 (i.e., three field times later), and the target letter at Field Time 5 (i.e., the prime duration was three times the duration of the backward mask, as in Experiment 2). The _ character of the set used by the interactive activation model was used as a backward mask in the simulation trials. When this character is input into the model, none of the abstract feature and letter detectors is activated. The idea was that, as with the checkerboard mask used in our experiment, the mask should prevent simple energy integration between prime and target (e.g., afterimage) without preventing integration of information at higher levels (e.g., fea- ture and abstract letter detectors) by actively inhibiting and/or exciting some of the feature units (e.g., by use of the \# character, which in the model corresponds to the superimposed features of $\mathrm{O}$ and $\mathrm{X}$ ). The stimuli used in the simulation were those of Experiment 2: We used (E, G, I, O, and $U$ ) as target letters, together with their similarity partners $(F, C, T, Q$, and $V)$ as primes. Table Al compares the results of two simulations with the empirical RT data obtained in the first three conditions of Experiment 2. The simulation data given in Table Al are based on mean values for the five trials run in each condition (i.e., one trial for each target letter). In Condition DIS, the letter prime for a given target was chosen at random among the other target and prime letters, excluding its similarity partner. Figure A1 illustrates the time course of activation of the $O$ detector for the three simulated priming conditions.

(Manuscript received February 9, 1990; revision accepted for publication August 8, 1990.) 